\title{
Reconstruction d'une lyre antique
}

\section{Annie Bélis}

\section{OpenEdition}

Journals

Édition électronique

URL : https://journals.openedition.org/ethnomusicologie/2342

ISSN : 2235-7688

\section{Éditeur}

ADEM - Ateliers d'ethnomusicologie

Édition imprimée

Date de publication : 1 janvier 1989

Pagination : 203-216

ISBN : 2-8257-0178-5

ISSN : 1662-372X

\section{Référence électronique}

Annie Bélis, « Reconstruction d'une lyre antique », Cahiers d'ethnomusicologie [En ligne], 2 | 1989, mis en ligne le 15 septembre 2011, consulté le 21 septembre 2021. URL : http://journals.openedition.org/ ethnomusicologie/2342 


\title{
RECONSTRUCTION D'UNE LYRE ANTIQUE
}

\author{
Annie Bélis, avec la collaboration de Jean-Claude Condi
}

Dans l'Antiquité grecque, l'instrument à cordes le plus populaire a toujours été la lyre: c'est avec elle que les enfants s'initiaient à la musique; dès qu'ils savaient lire, ils se rendaient chez un «cithariste» qui n'était pas, contrairement à ce que l'on pourrait croire, un professeur de cithare, mais un professeur de $\operatorname{lyre}^{1}$ : d'innombrables coupes à figures rouges représentent des scènes d'école où les enfants, le plus souvent assis en face du maître, tiennent une lyre et s'efforcent de reproduire l'exercice qui leur a été montré (fig. 1). Dans le Protagoras, Platon résume l'éducation musicale que recevaient les jeunes Athéniens:

"Quand l'élève sait jouer de son instrument, son maître lui fait connaître des oeuvres d'autres bons poètes, celles des compositeurs lyriques, qu'il lui fait exécuter sur la lyre $»^{2}$.

Seuls les individus les plus réfractaires à la musique se montraient incapables de se pénétrer de cet enseignement musical; c'est ainsi qu'Aristophane ironise sur «l'éducation de pourceau» du démagogue Cléon, qui, aux dires de ses anciens camarades d'école, ne savait accorder sa lyre qu'en doristi, et se refusait à apprendre un autre accord ${ }^{3}$. A côté de la cithare (fig. 2), dont se servaient

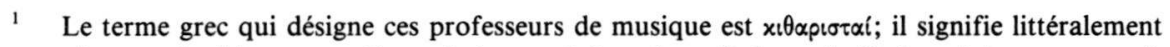

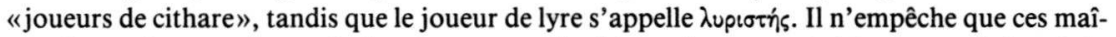
tres de musique enseignent la lyre, et non pas la cithare; le terme générique pour le jeu de la lyre reste $x ı \theta \alpha ́ p ı \sigma \mu \alpha$. Confirmation en est donnée par les très nombreuses représentations figurées d'écoles de musique: élèves et professeurs y jouent de la lyre.

2 Platon, Protagoras 326 a.

3 Aristophane, Cavaliers, v. 985; il s'agit en réalité d'introduire un jeu de mot sur l'accord de

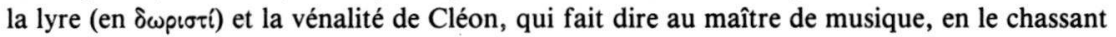
de son école, que «cet enfant n'est capable d'apprendre rien d'autre que le Dôrodokisti» (mot

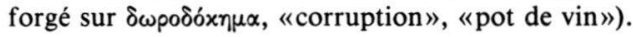


les professionnels dans les concerts et les compositions musicales, soit en solo instrumental (citharistique), soit en citharôdie (le musicien chantait en s'accompagnant), la lyre était un instrument pour amateurs: on en jouait dans les banquets, dans les fêtes privées, au gynécée. Son usage est attesté dès l'époque homérique: l'un des textes les plus anciens qui en parle est l'Hymne homérique à Hermès. Il raconte comment Hermès enfant invente la lyre: ramassant une carapace de tortue, il y adapte deux montants qu'il réunit par une traverse, tend une peau de bœuf sur la caisse formée par la carapace, et attache sept cordes sur l'instrument ainsi formét. La tradition d'une lyre construite sur les mêmes principes fondamentaux se perpétuera de l'époque archaïque jusqu'au déclin de l'empire romain: les écrivains en parlent, les peintres et les sculpteurs la figurent, les théoriciens en dissertent.

Dans ces conditions, il semblait possible de tenter de reconstruire une lyre d'époque classique ( $\mathrm{V}^{\mathrm{e}}$ siècle avant notre ère), en utilisant l'ensemble des sources disponibles. Une telle entreprise a été tentée par H. Roberts (1974, 1981: 303-13), dans le souci de reconstituer la technique de jeu des instruments à cordes. Pour ma part, je voulais essentiellement réaliser un instrument qui fût, autant que faire se peut, l'exacte réplique de ce qu'il était il y a vingt-cinq siècles. Pour ce faire, il convenait de réunir deux conditions préalables: tout d'abord, faire le bilan de l'ensemble des sources antiques relatives à la lyre (sources écrites, sources figurées, sources archéologiques), de manière à accumuler le plus grand nombre possible d'informations quant aux dimensions, aux matériaux, à la forme, à l'accord, bref, à la facture de l'instrument. La seconde condition était qu'au terme de ce premier travail, la proportion des incertitudes ou des inconnues n'oblige pas le luthier à inventer plutôt qu'à mettre en pratique les indications fournies par les sources antiques. En entreprenant cette reconstitution, nous savions, Jean-Claude Condi et moi, qu'il interviendrait nécessairement une part de conjecture, pour tous les points sur lesquels les sources se seraient avérées insuffisantes ou inexistantes. Cependant, il était convenu que toute solution technique envisagée serait considérée comme acceptable, dans la seule mesure où elle serait compatible avec les réalités antiques, et plus particulièrement avec les moyens techniques de l'époque. Inversement, serait exclue toute solution techniquement satisfaisante mais en contradiction avec une source, figurée ou autre, de l'époque concernée.

A ce jour, deux lyres ont été construites, et une troisième est en cours d'achèvement. La première est un «prototype», dont les caractéristiques sont

\footnotetext{
Hymne homérique à Hermès, v. 39-50: Après avoir ramassé dans la cour une tortue de montagne, Hermès nouveau-né rentre dans la maison: «alors, retournant la bête, avec un burin de fer mat il arracha la moëlle de vie à la tortue des montagnes [...]. Il tailla des tiges de roseau à la juste mesure, et les fixa en traversant dans le dos l'écaille de la tortue. Puis, avec l'intelligence qui est la sienne, il étendit sur le pourtour une peau de bœuf, adapta deux bras joints par une traverse, et tendit sept cordes harmonieuses en boyau de brebis» (Trad. Jean Humbert). Un récit analogue de l'invention de la lyre par Hermès se lit chez Lucien, Dialogue des Dieux, 4,223 , à ceci près que des chevilles sont adaptées à l'instrument.
} 


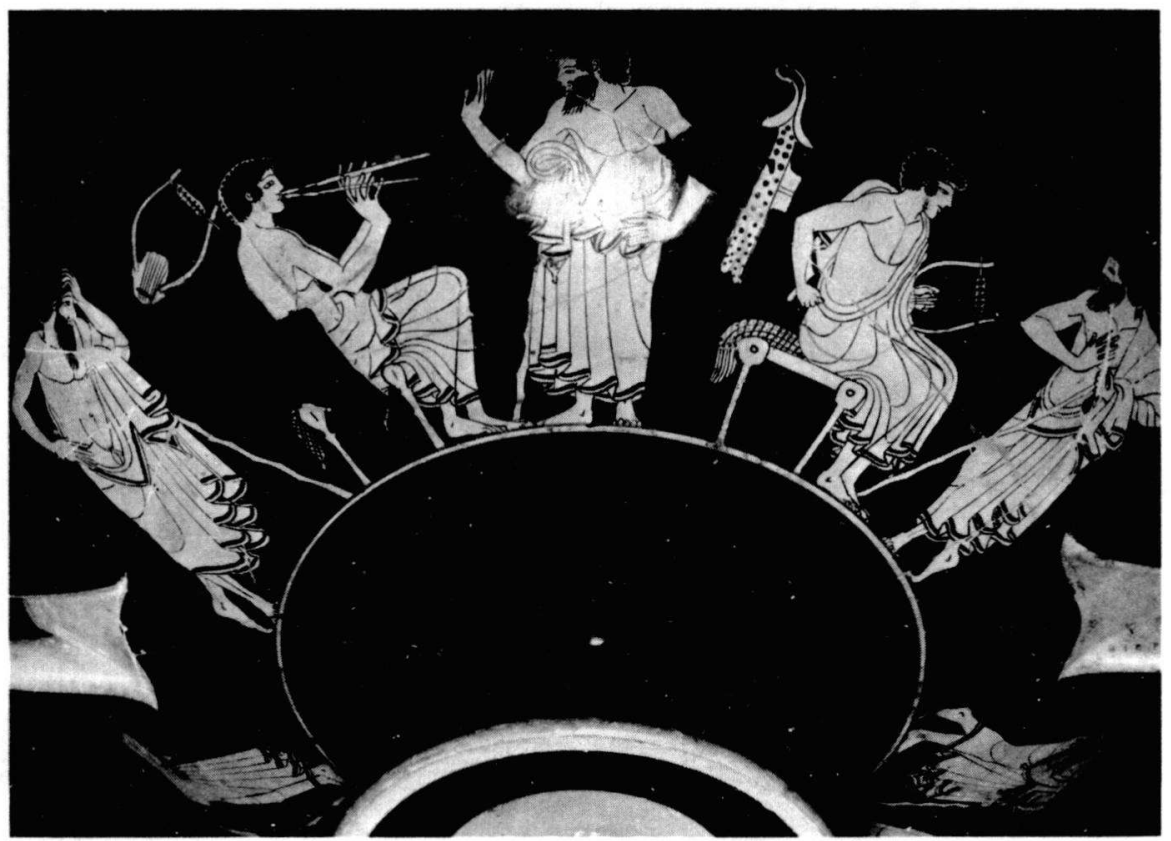

Fig. 1: Leçon de musique; coupe à figures rouges signée du potier Hiéron (Vienne, Kunsthistorisches Museum). Photo: Udo F. Sitzenfrey.

conformes aux modèles antiques, mais dont les finitions restaient imparfaites. La deuxième et la troisième versions ont rempli deux fonctions: d'abord une amélioration technique de certains aspects, ensuite l'introduction de variantes (dans le matériau, dans la forme des chevilles, etc.). La fig. 3 présente la lyre $\mathrm{N}^{\circ} 2$.

Le but du présent article n'est pas de décrire étape par étape la fabrication de l'instrument. Il se place en quelque sorte en amont; il s'agit ici de montrer de quelle manière ont été exploitées les différentes sources antiques relatives à la lyre, en mettant en relief les limites ou les difficultés auxquelles on se heurte. Méthodologiquement, l'exploitation de ces sources est paradoxale. En effet, alors qu'on possède un grand nombre de vestiges d'instruments à vent (auloi grecs et tibiae romaines) ${ }^{5}$, ce qui devrait en assurer la reconstitution dans de bonnes conditions, aucun instrument à cordes grec n'a été mis au jour dans des fouilles ${ }^{6}$. Un seul exemplaire de lyre est parvenu jusqu'à nous, la «lyre Elgin", du nom du diplomate britannique qui la ramena en Grande-Bretagne. L'instrument se trouve aujourd'hui au British Museum (fig. 4); ses bras et sa traverse sont d'origine; rien ne subsiste des cordes, ni de la peau, ni des chevilles;

5 J'ai répertorié et étudié entre cinq et six cents fragments d'instruments de ce type; seuls un petit nombre d'entre eux ont été publiés (Bélis 1985b: 8-15; 1988: 109-18; 1989).

6 A l'exception de la lyre Elgin (qui n'est pas complète) et des vestiges d'une éventuelle autre lyre, également rapportée de Grèce par lord Elgin (Pöhlmann 1988: 95-107), et elle aussi au British Museum. 


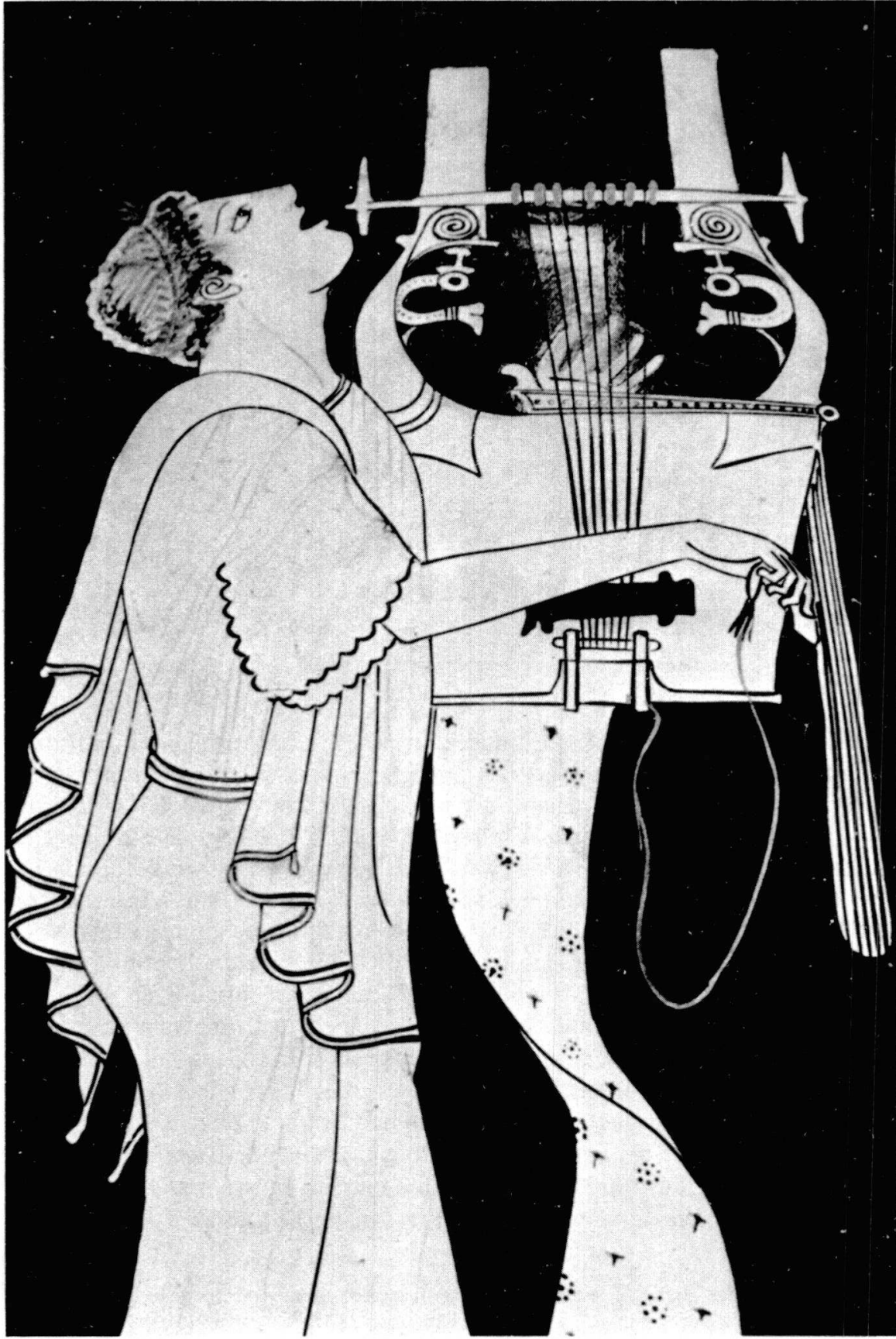

Fig. 2: Citharôde; amphore du Peintre de Berlin, ca. 490 av. J.C. (New York, Metropolitan Museum of Art, $\mathrm{N}^{\circ}$ 56.171.38). Photo: $\mathrm{X}$. 


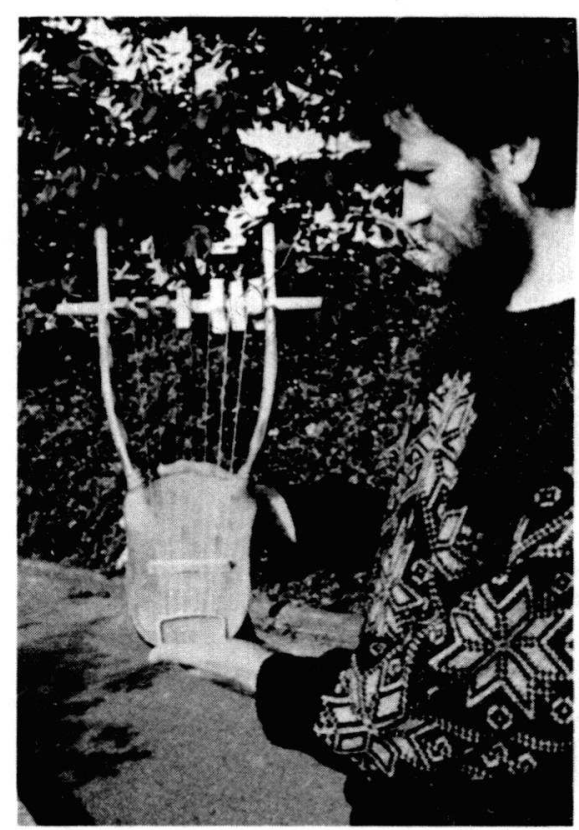

Fig. 3: Lyre réalisée par Jean-Claude Condi (prototype). Photo: G.J. van Kooten.

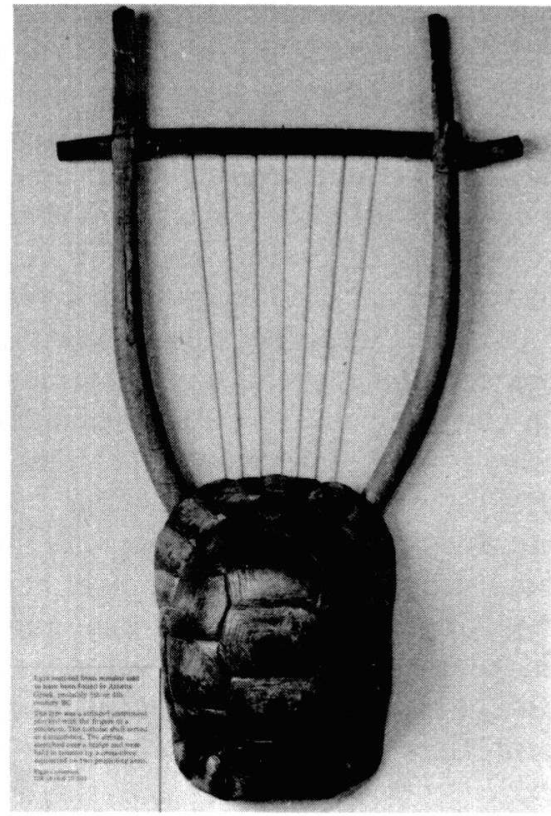

Fig. 4: «Elgin Lyre» (Londres, British Museum, $\mathrm{N}^{\circ}$ GR 1816.6-10.501). Photo du Musée.

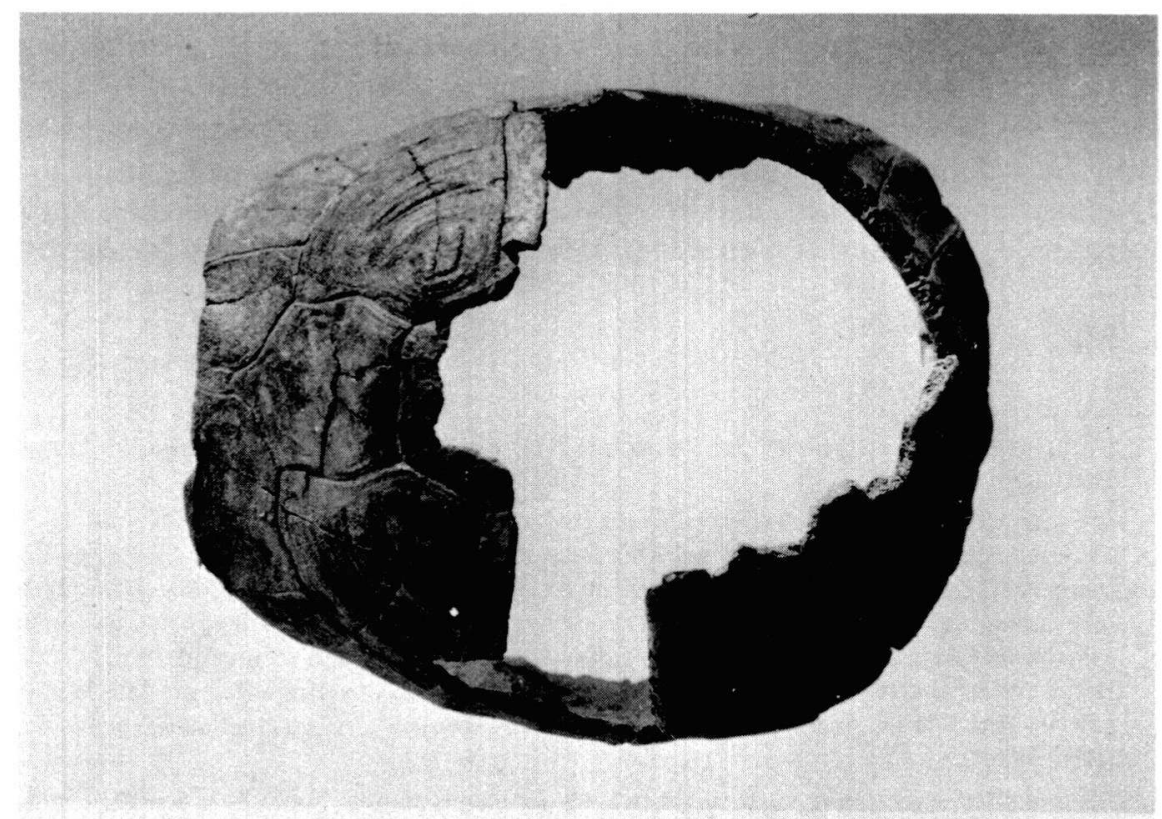

Fig. 5: Carapace de tortue d'Argos, inv. A 56 (U.14) (Musée d'Argos). Photo: E.F.A. 
quelques fragments de la carapace antique ont été retrouvés, trop fragiles pour être exposés avec l'instrument. On ne connaît pas précisément les circonstances de sa découverte, ce qui laisse planer quelque doute, aux yeux de certains, sur son authenticité: je ne partage pas ces réserves ${ }^{7}$. Outre cette lyre, nous possédons encore une demi-douzaine de carapaces de tortues ayant servi de caisse à des lyres antiques - mais dont aucun autre élément n'a survécu. Ces carapaces sont sciées, évidées, et percées de petits trous, ce qui atteste qu'elles ont été travaillées par des fabricants de lyres (Phaklaris 1977, Courbin 1980). La fig. 5 présente l'une d'entre elles, découverte par $\mathrm{P}$. Courbin à Argos. Ces sources archéologiques sont donc relativement peu nombreuses, par comparaison avec les quelque cinq à six cents fragments d'auloi conservés dans les musées. Pourtant, avec ces seuls vestiges, il est plus facile de reconstituer une lyre qu'un aulos grec ou une tibia romaine: la lyre, tout d'abord, est un instrument nettement moins complexe que ne le sont les instruments à vent antiques; d'autre part, pour peu qu'on veuille examiner et interpréter comme il convient les monuments figurés, et en appeler aux sources écrites, il devient possible de restituer en théorie une lyre-type (les variantes étant somme toute peu nombreuses), tandis que cette tâche est quasiment irréalisable pour les auloi. Car chaque aulète professionnel se faisait fabriquer sur commande ses instruments, formulait ses exigences et ses souhaits au facteur, de sorte qu'il n'y a pas deux auloi qui soient rigoureusement semblables ${ }^{8}$. Dans ces conditions, on peut espérer fabriquer aujourd'hui une reconstitution de lyre antique qui sera également un fac-simile de la lyre Elgin, par exemple, mais il est sûr qu'on ne pourra réaliser, au mieux, qu'un fac-simile d'un aulos particulier, et non pas reconstituer un aulos-type'. Au demeurant, on dispose d'un atout supplémentaire pour reconstruire une lyre, ce dont on est dépourvu pour les instruments à vent: c'est le relatif conservatisme des fabricants antiques qui, pendant des siècles, ont préservé les traditions. Sans doute cette permancence s'explique-t-elle par le caractère populaire d'un instrument à la fois modeste et peu coûteux, dont on ne cherchait pas à accroître les capacités. Il n'en alla pas de même pour les cithares de concert,

7 La «lyre Elgin» a été publiée par Reinach (1911), Roberts (1981), Bélis (1985a: 213-214) et Pöhlmann (1988).

8 C'est ce qui ressort de deux témoignages catégoriques: Platon, République X, 601 D-E: «L'aulète renseigne le fabricant d'aulos sur les auloi qui lui servent à jouer, et c'est lui qui dira comment il faut les construire, et le fabricant lui obéira»; Plutarque, De la Musique, 1138 a: «Téléphane de Mégare avait une telle aversion pour [le mécanisme des] syringes qu'il ne permit jamais aux fabricants d'en adapter à ses auloi". Il n'y a pas lieu de s'étonner, dans ces conditions, que les quatre grandes tibiae en argent et en bronze, découvertes à Pompéi dans la maison de Gaius Vibius, soient très différentes les unes des autres, tout en étant du même facteur (Bélis 1989).

9 Pour réaliser ce fac-simile, on se heurtera à des difficultés considérables, dues essentiellement à la complexité des mécanismes et à l'ignorance relative où nous sommes des types d'anches à utiliser. 
ni pour les auloi, dont les prix atteignirent des fortunes ${ }^{10}$. Tout au plus voit-on, à l'époque romaine, des lyres dont la caisse, au lieu d'être faite en carapace de tortue, est en marqueterie de bois précieux ${ }^{11}$, pour céder au goût de luxe affiché par certains particuliers. L'évolution la plus sensible touche à deux éléments principaux: les chevilles et les bras.

Le système le plus couramment employé, pour les bras, était d'utiliser deux rameaux de bois; la céramique attique à figures rouges (style de peinture qui fleurit durant tout le $\mathrm{V}^{\mathrm{e}}$ siècle av. J.C.) (fig. 6-11) et les sources écrites ne laissent pas de doute à ce sujet. Théophraste, dans son Histoire des Plantes (V, 7, 6), précise même que les bras des lyres étaient construits avec du bois de chênekermès, un bois dur et noir, dont on faisait aussi des pièces de brouettes, tant il est solide. Dans certains cas, on se servait de cornes d'antilopes, dont les élégantes courbures donnaient quelque beauté supplémentaire à un instrument par ailleurs très simple: les Romains semblent avoir privilégié ce choix, comme le montre, entre autres, la belle fresque d'Achille et du centaure Chiron ${ }^{12}$. Il n'est pas exclu que les fabricants de lyres aient imité ces cornes, comme ils imitaient les carapaces de tortues. En Grèce, le fait a dû rester plus rare; c'est ce qu'incite à penser une remarque de l'historien Hérodote, qui signale comme une curiosité un certain type de lyre "phénicienne», dont les bras sont faits avec les cornes de l'oryx (une sorte d'antilope) libyen ${ }^{13}$.

Quant aux chevilles, plusieurs systèmes furent utilisés. Dans l'Hymne homérique à Hermès, rien n'est dit d'un quelconque mécanisme: le petit dieu entortille les cordes autour du joug. C'est un autre texte homérique qui mentionne le plus ancien système de «chevilles», qu'on appelle xó $\lambda \lambda \circ \psi$ : la corde est fixée sur la traverse autour d'un morceau de cuir pris dans la nuque d'un bœuf $^{14}$. Cette dernière information ne provient pas de l'Odyssée, mais d'une notice qu'un lexicographe d'Alexandrie, Hésychius, qui vivait au $\mathrm{V}^{\mathrm{e}}$ siècle de notre ère, a écrite pour expliquer un terme technique, $x o ́ \lambda \lambda \alpha \beta o \varsigma$, et dans laquelle il décrit son usage dans la lyre et lui donne un synonyme: xó $\lambda \lambda \circ \psi^{15}$. Cette incursion un peu plus précise dans les sources écrites nous fournit l'occasion d'une remarque sur leur exploitation. Il est rare que l'on puisse directement tirer parti d'un témoignage littéraire sur les instruments de musique et leur facture, pour la simple raison que son auteur reste allusif, ou emploie des termes qui ne sont pas souvent descriptifs. Aussi faut-il ici passer par des

10 Un aulète thébain très célèbre dans toute l'Antiquité, Isménias, qui vécut dans le dernier tiers du IV ${ }^{\text {e }}$ siècle av. J.C., acheta des auloi à un fabricant corinthien pour la somme fabuleuse de sept talents (42000 drachmes), alors qu'une trompette ne valait que soixante drachmes (Lucien, Le collectionneur de livres 5).

11 Pline le Jeune, Histoire Naturelle, XI, 124.

12 Fresque d'Herculanum, ca. 75 ap. J.C., Naples, Museo Nazionale, N 9109.

13 Hérodote, IV.192; cf. Athénée, citant Sémos de Délos, XIV, 637 b.

14 Cf. ci-dessus, note 4.

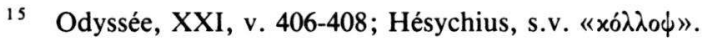




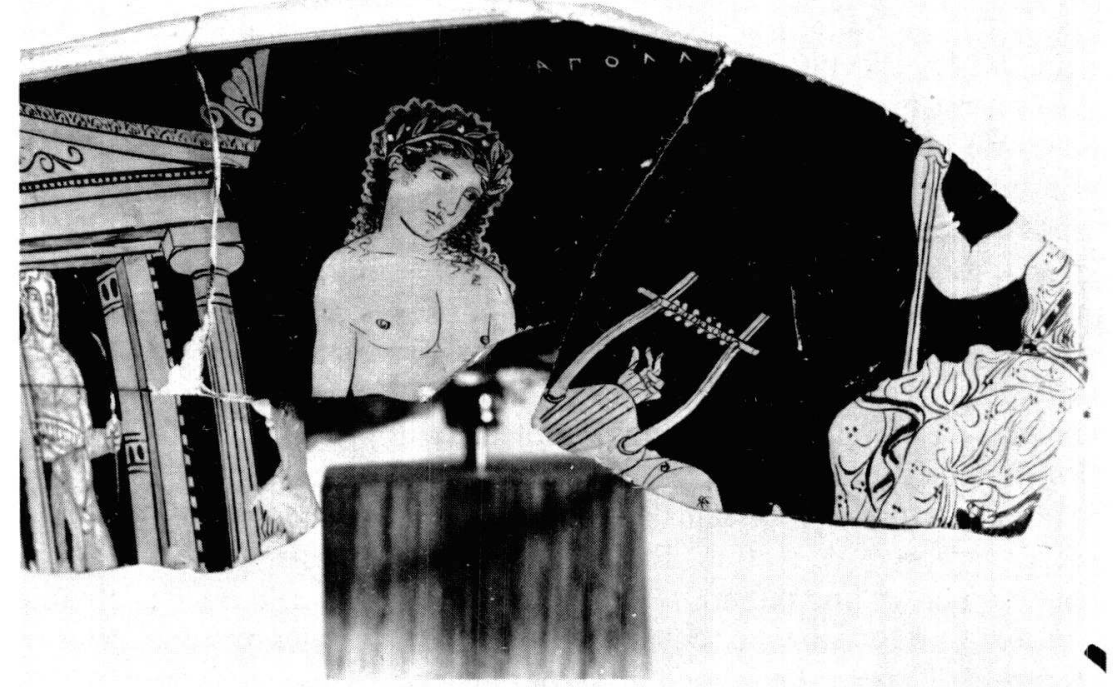

Fig. 6: Fragment de cratère en calice du Peintre de la Naissance de Dionysos (410/400 av. J.C.) (Amsterdam, Musée Allard Pierson, $\mathrm{N}^{\circ} 2579$ ). Photo du Musée.
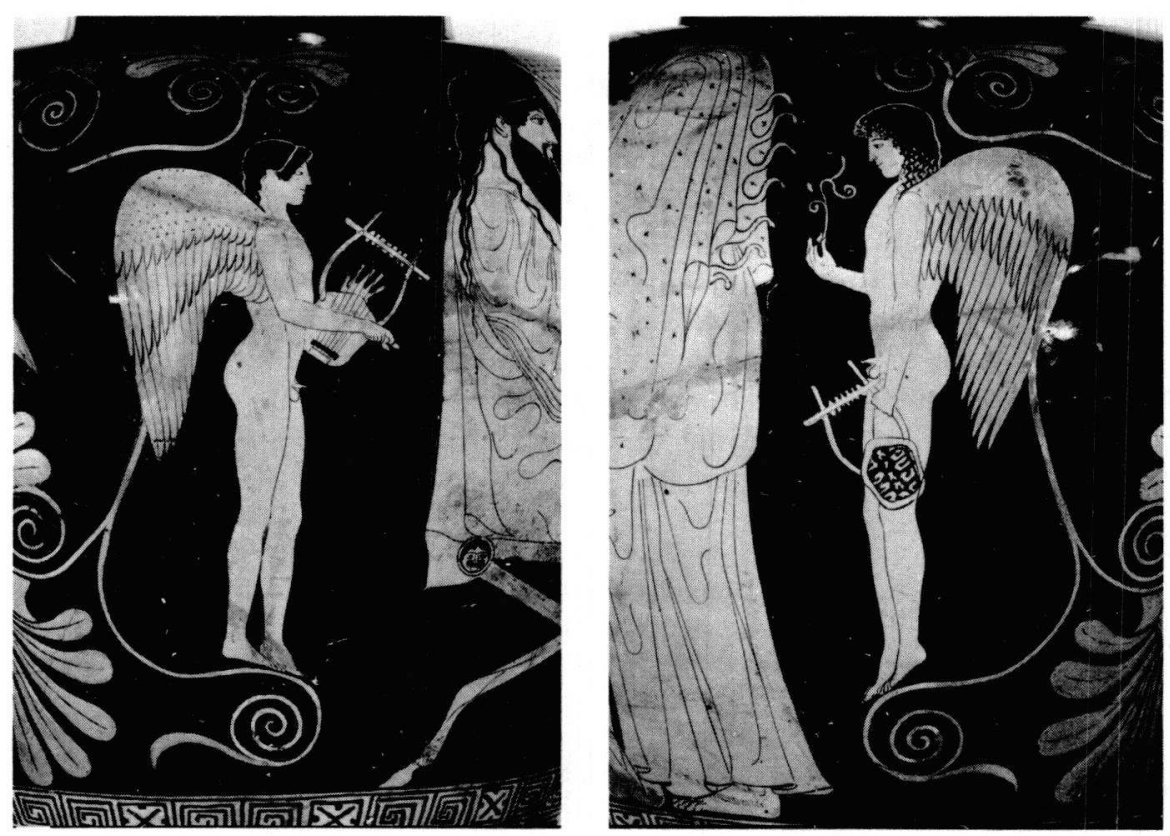

Fig. 7-8: Stamnos à figures rouges du Peintre de Munich, 2413 (München, Museum Antiker Kleinkunst, $\mathrm{N}^{\circ}$ J 345). Photo du Musée. 


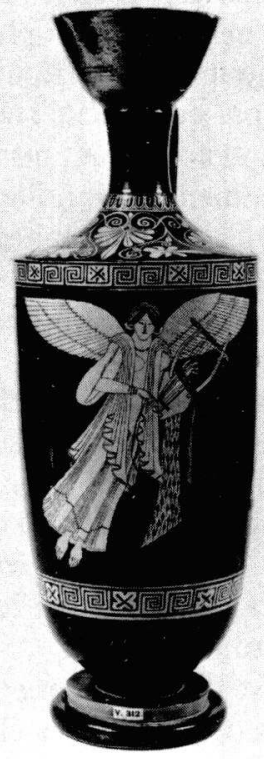

Fig. 9: Lécythe à fond noir du Peintre de Pan (ca. 490 av. J.C.), figurant Niké ailée à la lyre (Oxford, Ashmolean Museum, $\mathrm{N}^{\circ}$ 312). Photo du Musée.

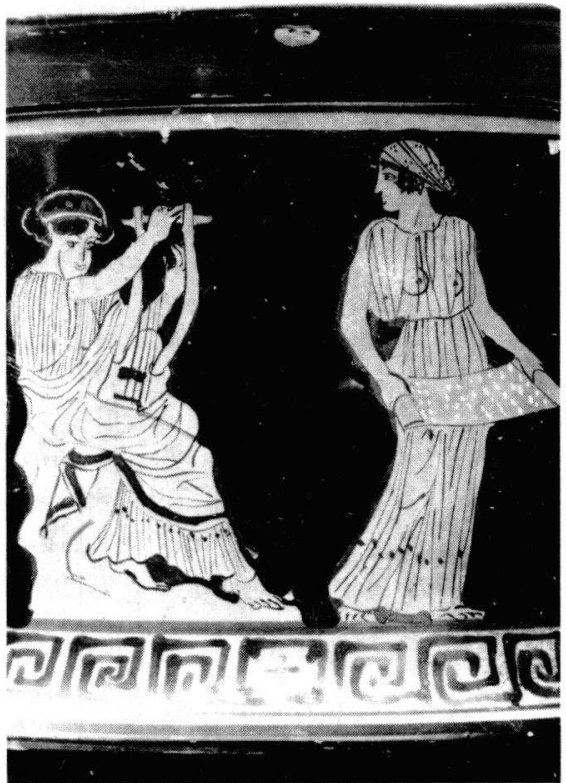

Fig. 10: Pyxis MN 1241 (Athènes, Musée National). Photo du Musée.

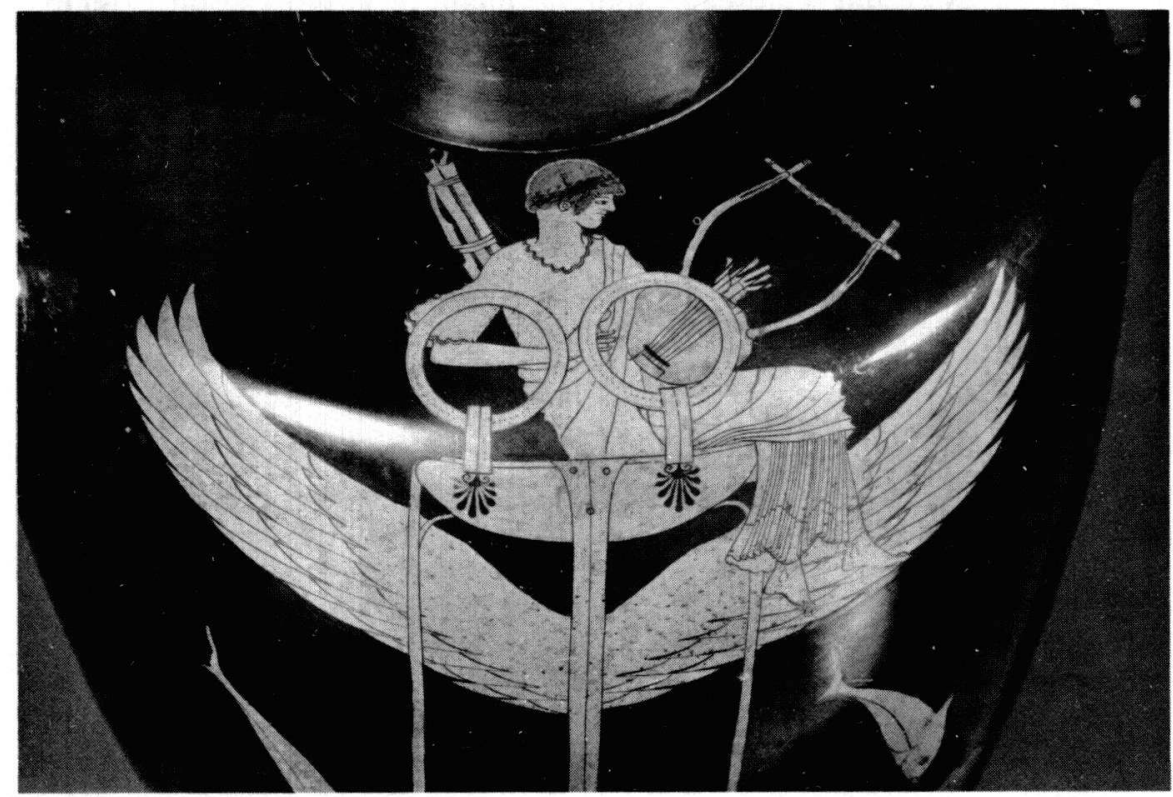

Fig. 11: Hydrie à figures rouges du Peintre de Berlin (ARV[2] 209.166) (Museo Gregoriano, Vatican). Photo: Musei Vaticani. 
intermédiaires beaucoup plus tardifs pour l'éclairer. Or, tel était justement le rôle des lexicographes: expliciter des mots que l'on ne comprenait plus. L'utilisation de ces précieuses notices ne se fait pas toujours sans difficultés. Étant donné que ces érudits, certes fins lettrés, n'étaient pas des spécialistes de musique, étant donné aussi que la tradition manuscrite qui nous a fait parvenir leurs textes n'est pas exempte d'erreurs, il arrive couramment qu'il faille mettre en doute leurs informations. Ici, il n'y a pas à le faire: les plus anciennes représentations de lyres (ou de phorminx) viennent confirmer l'existence de ce premier et rudimentaire système d'attache des cordes autour de bourrelets de cuir (Bélis 1985a: 217-19).

Le second mécanisme, que l'on peut désigner par le terme français de «cheville», est attesté à la fois par les représentations figurées et par les témoignages littéraires ou lexicographiques. Grâce à la céramique, on peut fixer la date de cette innovation vers la moitié du $\mathrm{V}^{\mathrm{e}}$ siècle avant notre ère. Au lieu d'enrouler les cordes autour du joug sur des tampons de cuir, on les fait passer dans des pièces adaptées à la traverse, que les auteurs grecs désignent par le terme de $\pi \alpha \sigma \sigma \alpha \dot{\lambda} \iota \sigma x \iota^{16}$ : c'est le diminutif de $\pi \dot{\alpha} \sigma \sigma \alpha \lambda \circ \varsigma$, «clou» ou «piquet». Sans doute est-ce ce système qui apparaît sur la petite lyre votive de Heidelberg (l'objet est en bronze) (fig. 12). Pour notre part, nous avons opté pour des chevilles de bois $^{17}$, fichées dans le joug, qui correspondent assez exactement à l'ensemble des indications fournies par les meilleures représentations figurées: le fragment de cratère à figures rouges du Musée Allard Pierson d'Amsterdam, d'une précision extrême (fig. 6), œuvre du Peintre de la Naissance de Dionysos (ca. 410/400 av, J.C.), qui représente Apollon jouant de la lyre devant le temple de Delphes; de même, le très fameux «Trône de Boston» (fig. 13), qui date du $\mathrm{V}^{\mathrm{e}}$ siècle, et le lécythe à fond noir du Peintre de Pan (ca. 490 av. J.C.) de l'Ashmolean Museum d'Oxford (fig. 9). La fig. 14a donne le schéma des chevilles fabriquées par J.Cl. Condi, et la fig. 14b indique le trajet de la corde autour du joug et de la cheville (il s'agit du système définitif; celui qui a été utilisé sur le prototype a été corrigé pour mieux correspondre aux représentations figurées).

Abondantes et définitives sur certains points, les sources écrites font totalement défaut sur d'autres, en particulier sur tout ce qui se rapporte aux techniques de fabrication et à l'assemblage des parties de l'instrument. Dans ce domaine, nous n'avons pu exploiter que les représentations figurées et nous conformer qu'à ce que la lyre Elgin en laissait deviner. D'une manière générale, J.Cl. Condi a pris le parti de travailler avec des techniques qui ne faisaient

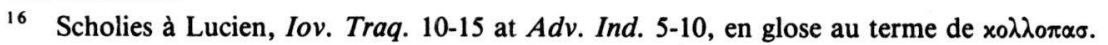

17 A l'époque romaine, seuls les instruments simples garderont des chevilles en bois, à en juger par le témoignage de Lucien, Le collectionneur de livres, 8: au concours pythique se présentent deux citharôdes; le premier, Evagelos de Tarente, est richement vêtu, et joue d'une cithare en or, ornée de pierres précieuses mais se révèle mauvais musicien; un autre, Eumèlos d'Élis, mal habillé, et jouant d'une "vielle cithare à chevilles de bois», remporte le concours. 


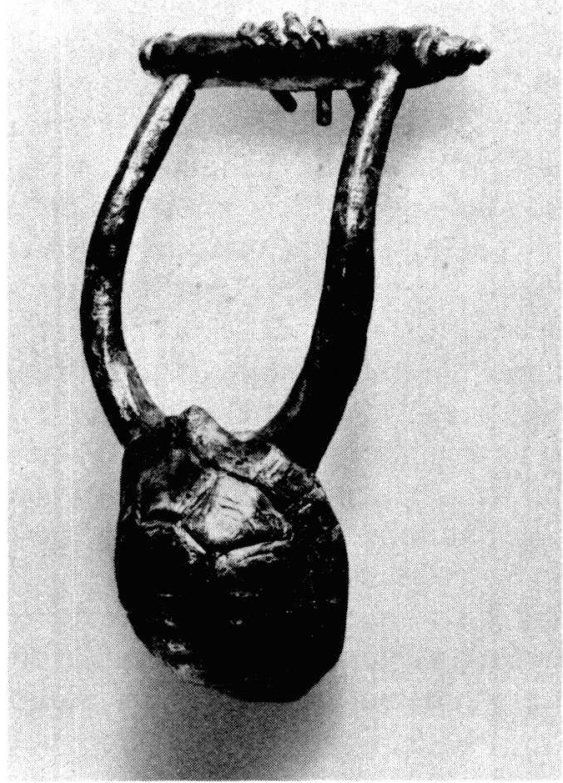

Fig. 12: Lyre votive en bronze du Musée de Heidelberg, inv. $\mathrm{N}^{\circ} 76 / 2$. Photo: Archäologisches Institut der Universität Heidelberg.

a.

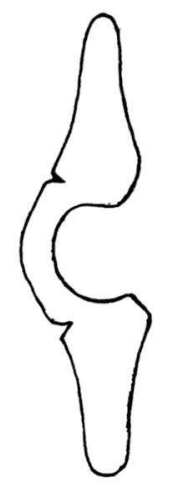

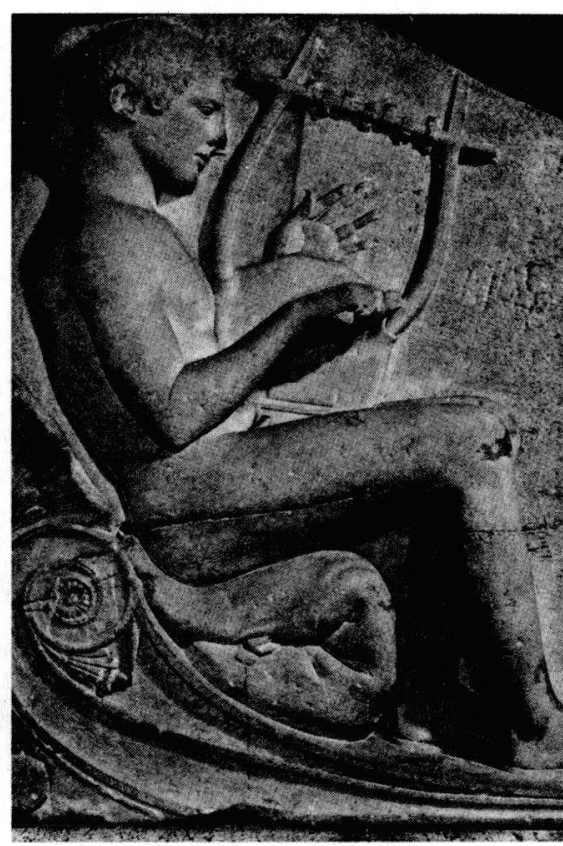

Fig. 13: "Trône de Boston» (New York, Metropolitan Museum of Art). Photo: X.

Fig. 14a: Schéma d'une cheville (profil) de la lyre $\mathrm{N}^{\circ} 2$ de J.-Cl. Condi; Dessin: A. Bélis.

Fig. 14b: Trajet de la corde autour de la cheville et du joug. Dessin: J.-Cl. Condi et A. Bélis.

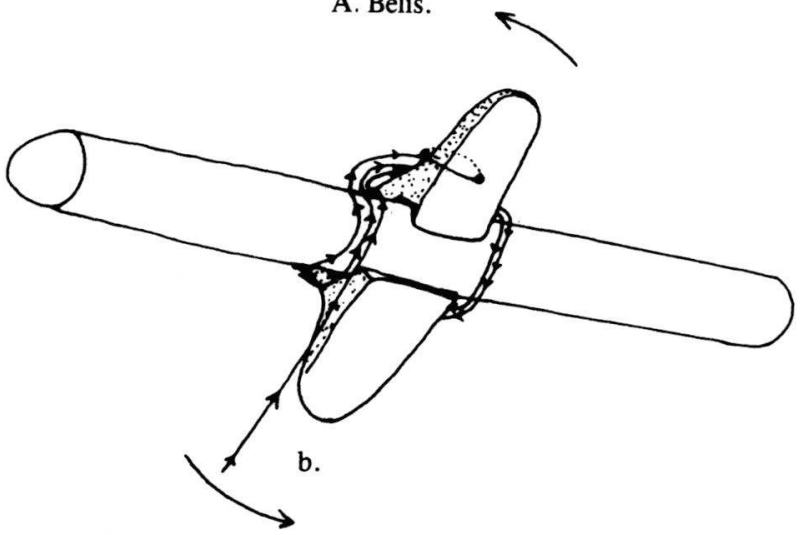


appel à aucun procédé industriel, pas même pour réaliser le cintrage des montants.

Dans leur très grande majorité, les peintres de vases grecs ont représenté des lyricines de profil, ou de trois-quarts, de telle manière que l'instrument est vu de face (fig. 6-9 et 11). Il arrive parfois que les peintres aient essayé de dessiner les visages de face, et de présenter la lyre sous un angle inhabituel (de troisquarts, sur la fig. 10). Autant l'incurvation latérale des bras à la sortie de la caisse est bien rendue dans la plupart des cas, autant le cintrage d'avant et arrière leur a causé d'insurmontables difficultés. Tout au plus ont-ils réussi à le suggérer, sans vraiment parvenir à le représenter fidèlement en même temps que l'incurvation latérale. Aussi était-il impossible de résoudre cette question à l'aide des seules représentations figurées; pas davantage ne pouvions-nous trouver de réponse avec la lyre Elgin: le bois des montants s'est délité, et rien ne subsiste plus du deuxième cintrage. L'instrument repose aujourd'hui à plat sur son présentoir. C'est la petite lyre votive en bronze du Musée de Heidelberg qui nous a servi de modèle pour ces deux opérations (fig. 12).

Comment fallait-il assembler les bras et la traverse? Les meilleures représentations figurées (fig. 6 et 13) nous avaient appris que le montant, après avoir rencontré la traverse, n'était plus de section circulaire, mais semi-circulaire. En outre, juste au point de contact avec la traverse, le montant semble faire une sorte de repli arrondi, comme pour en épouser le contour. Quant au type d'encastrement dont nous avons doté nos lyres, il est rigoureusement conforme à celui de la lyre Elgin, que j'ai détaillé dans un article précécdent (Bélis 1985: 215). Son avantage principal est d'être d'autant plus solide que la tension à laquelle il est soumis est plus grande.

Il est un détail qui mérite qu'on s'y arrête un instant. Bon nombre de représentations figurées de lyre, et tout spécialemet les plus précises, ont un point commun qui pourrait passer inaperçu: un trait noir, dessiné sur la moitié supérieure du montant, jusqu'à sa rencontre avec la traverse. Comment l'interpréter? S'agit-il seulement d'une décoration peinte, d'un filet, ou d'une rainure qui aurait quelque utilité? En réalité, comme le démontrent, et les reliefs et la lyre Elgin, ce que les peintres rendent par un trait de pinceau est une étroite nervure en très léger relief. Avant la fabrication de la lyre, nous n'avions pas trouvé de justification précise à ce détail que nous avions convenu, cependant, de restituer exactement. L'explication s'est révélée au cours de la construction du montant: pour dégager la butée, il est nécessaire d'atteindre le cœur du bois, puis de le faite disparaître. Cette opération réalisée, il devient tout naturel de remplir le trou ainsi obtenu par une pièce (en l'occurrence, en os), qui vient à la fois répondre à un besoin mécanique et satisfaire à une exigence esthétique.

L'accord de cette lyre, dont les sept cordes sont en boyau de mouton (conformément aux indications textuelles), est de deux tétracordes conjoints en genre chromatique: tel est le système le plus utilisé au $\mathrm{V}^{\mathrm{e}}$ siècle avant notre ère. La lyre $N^{\circ} 3$ sera, elle, octocorde, avec deux tétracordes disjoints couvrant une octave. La justesse peut être corrigée dans les limites d'un seul quart de tour des chevilles (au-delà, la corde viendrait à se rompre). Pour compléter 
l'instrument et en permettre le jeu dans les mêmes conditions que les lyricines grecs, un baudrier de cuir a été attaché à la base du montant le plus éloigné de l'instrumentiste qui se le passe autour du poignet gauche et tient ainsi plus solidement son instrument. Un plectre, dont les dimensions peuvent nous paraître aujourd'hui démesurées, mais qui respectent celles de l'époque (fig. 13), est en cours de fabrication. On sera ainsi en mesure d'utiliser les deux techniques de

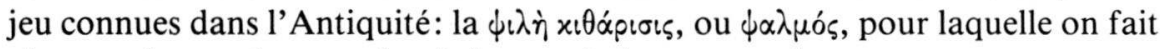
résonner les cordes avec les doigts, et le jeu «avec plectre».

Comme on le sait, l'Antiquité gréco-romaine nous a transmis une cinquantaine de partitions, qui sont essentiellement des papyrus. La plupart sont écrites en notation vocale (il s'agit donc de mélodies sur lesquelles on chantait des textes poétiques), mais un certain nombre sont en notation instrumentale. Peut-on espérer jouer sur la lyre reconstituée telle ou telle de ces partitions, dont la plus ancienne est du $\mathrm{III}^{\mathrm{e}}$ siècle avant notre ère? Pour ce faire, il sera nécessaire de résoudre le problème majeur auquel se heurtera notre entreprise: trouver douze notes sur un instrument qui ne comporte pas de manche, et qui ne possède que sept ou huit cordes.

En l'état actuel de la question, considérons déjà pour acquis que la lyre telle que nous l'avons réalisée, est aussi proche que faire se peut de ses prédécesseurs antiques, de telle manière que se trouvent réunies les conditions optimales pour cette expérimentation musicale.

\section{Bibliographie}

BÉLIS Annie

1985a «A propos de la construction de la lyre». Bulletin de Correspondance Hellénique 109: 201-20.

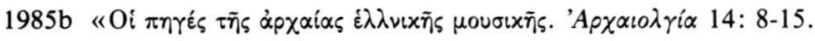

1988 "Charnières ou auloi?» Revue Archéologique 1: 109-118.

1989 «Studying and dating ancient Greek auloi and Roman tibiae: a methodology». In: Ellen Hickmann \& David Hughes, Ed. The Archaeology of Early Music Cultures, Third International Meeting of the ICTM Study Group on Music Archaeology. : Verlag für systematische Musikwissenschaft (p. 233-48).

COURBIN P.

1980 «Les lyres d'Argos». Bulletin de Correspondance Hellénique, Supplément VI, Etudes argiennes. Paris: De Boccard, p. 93-114.

PHAKLARIS P.

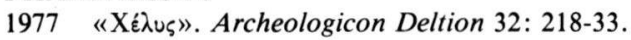


PÖHLMANN E.

1988 «Zwei Elgin-Leiern im British Museum? Beiträge zur antiken und neueren Musikgeschichte.» In: Quellen und Studien zur Musikgeschichte von der Antike bis in die Gegenwart 17. Frankfurt: Verlag Peter Lang, p. 95-107.

REINACH Th.

1911 Article «Lyra». In: Dictionnaire des Antiquités Daremberg-Saglio-Pottier. Paris. Hachette.

ROBERTS H.

1974 Greek stringed instruments, 700-200 B.C.. PhD Thesis, Reading University.

1981 «Reconstructing the Greek tortoise-shell lyre». World Archaeology 12(3): 303-13. 